\title{
Zero Power Non-Contact Suspension System with Permanent Magnet Motion Feedback*
}

\author{
Feng SUN** and Koichi OKA** \\ ** Kochi University of Technology \\ 185 Miyanokuchi, Tosayamada, Kami city, Kochi 782-8502, Japan \\ E-mail: oka.koichi@kochi-tech.ac.jp
}

\begin{abstract}
This paper proposes a zero power control method for a permanent magnetic suspension system consisting mainly of a permanent magnet, an actuator, sensors, a suspended iron ball and a spring. A system using this zero power control method will consume quasi-zero power when the levitated object is suspended in an equilibrium state. To realize zero power control, a spring is installed in the magnetic suspension device to counterbalance the gravitational force on the actuator in the equilibrium position. In addition, an integral feedback loop in the controller affords zero actuator current when the device is in a balanced state. In this study, a model was set up for feasibility analysis, a prototype was manufactured for experimental confirmation, numerical simulations of zero power control with nonlinear attractive force were carried out based on the model, and experiments were completed to confirm the practicality of the prototype. The simulations and experiments were performed under varied conditions, such as without springs and without zero power control, with springs and without zero power control, with springs and with zero power control, using different springs and integral feedback gains. Some results are shown and analyzed in this paper. All results indicate that this zero power control method is feasible and effective for use in this suspension system with a permanent magnet motion feedback loop.
\end{abstract}

Key words: Motion Feedback Loop, Zero Power Control, Magnetic Suspension, Permanent Magnet, Spring, Linear Actuator, Integral Feedback Loop

\section{Introduction}

Magnetic suspension systems have been extensively studied and have found numerous applications. Most magnetic suspension systems are electromagnetic suspension systems (EMS systems) that utilize electromagnets. A zero power control method for EMS systems has been proposed by Morishita and Azukizawa with the aim of reducing steady state energy consumption ${ }^{(1)}$, where this electromagnetic suspension system includes a permanent magnet and an electromagnet. When the device is suspended, the permanent magnetic force counterbalances the gravitational force acting on the object, and the electromagnetic force maintains the balance. Consequently, energy is saved. This zero power control method for EMS systems has been adopted by many researchers and used in various magnetic suspension systems. Misuno et al. have developed a vibration isolation system using this zero power magnetic suspension system that behaves as a spring capable of infinite negative stiffness ${ }^{(2)}{ }^{(3)}$. Ishino et al. have presented a method of increasing the load capacity of the magnetic suspension system with this zero power control method ${ }^{(4)}$. Kim et al. have 
presented a zero power control scheme using an extended Luenberger observer, taking into consideration nonlinear gap and current characteristics to improve the magnetic levitation performance ${ }^{(5)}$. Morishita and Itoh have proposed a self-gap-detecting zero power controller for an EMS system ${ }^{(6)}$. In that controller, a state observer evaluates the air gap lengths and their change rates with respect to time based on the currents and voltages exciting the magnet units. The exciting voltage compensator calculates compensation voltages to prevent interaction between the two control axes. The control parameters can be altered by varying the output of the coil resistance evaluator. Myounggyu et al. have proposed a virtual zero power (VZP) controller consisting of a standard linear-quadratic (LQ) regulator and a Kalman filter ${ }^{(7)}$. The controller is used in an axial bearing in a pediaflow ventricular assist device. The Kalman filter estimates both the equilibrium position to which the feedback controller drives the system, and the covariance of sensor noise and axial disturbance forces on the rotor, which is used to select LQ regulator optimal gains. In this way, the controller can bring the actuator force to zero.

Recently, some researchers have proposed permanent magnetic suspension systems that control the attractive force between a permanent magnet and a ferromagnetic floater. Misuno et al. have proposed a magnetic suspension system with a permanent magnet and three flux-path control modules consisting of a ferromagnetic plate, a voice coil motor (VCM), and a displacement sensor ${ }^{(8)}$. This system controls the attractive force of the magnet by changing the flux path with the flux-path control modules. Some of systems can also realize zero power control. Ueno and Higuchi presented a zero-power magnetic levitation technique using a composite of magnetostrictive and piezoelectric materials ${ }^{(9)}$. That technique utilized the capacitive property of the piezoelectric materials so that no current flowed in static operation, to realize the zero power control at any reference gap or load. Finally, Oka et al. proposed a magnetic suspension system with a permanent magnet and a rotary actuator that drove the permanent magnet varying magnetic flux path ${ }^{(10)}$. The suspension force was controlled through the variable magnetic flux path. That system resulted in a zero attractive force, changed the polarity of stator poles, and realized zero power control in an equilibrium state.

So far, we have proposed several kinds of permanent magnetic suspension systems with permanent magnets and linear actuators. A hanging type permanent magnetic system with a permanent and a VCM was proposed. In that system, non-contact levitation was realized by adjusting the magnetic force via the control of the air gap between the permanent magnet and a ferromagnetic ceiling using the $\mathrm{VCM}^{(11)}$. A 2-DOF (degrees of freedom) magnetically suspended manipulation system was proposed ${ }^{(12)-(14)}$. In that system, an iron ball was suspended and manipulated in two degrees. Moreover, a non-contact spinning mechanism was proposed to realize an iron ball rotating using remanent magnetization with permanent magnets and linear actuators ${ }^{(15)(16)}$.

In the present paper, a zero power control method is proposed for a 1-DOF magnetic suspension system consisting of a permanent magnet, a VCM, and a suspended iron ball. This system uses a permanent magnet, a linear actuator, and no electromagnet; as a result, there is no heat generation and it is easier to realize remote non-contact manipulation by using a long rod. However, when this system suspends an object with non-contact, the actuator has to support all the weight of the moving part and the suspended object. Therefore, the energy consumption becomes a significant problem. In order to solve this problem, a zero power control method is adopted to reduce the energy consumption. A spring is assembled in the device, and an integral feedback loop is used in the control system. Quasi-zero power is then consumed in the steady state.

First, the principle of the permanent magnetic suspension is explained. The realization of zero power control method is then analyzed in a prototype, through a model, and in the controller. Finally, some numerical simulation and experimental results are shown and 
discussed. All of the results indicate that this zero power control method is feasible and effective for use in this suspension system with a permanent magnet motion feedback loop.

\section{Principle of magnetic suspension}

The principle of this levitation system is explained in terms of the suspension system scheme shown in Fig. 1. The suspension system consists of an actuator, a permanent magnet, and a suspended object. The permanent magnet generates the suspension force, the actuator drives the magnet keeping the suspension stable, and the suspended object is a ferromagnetic body. When the object is suspended with non-contact in a steady state, the relationship between attractive force and gravitational force is as shown in Fig. 2. The levitation direction is vertical, and in the equilibrium position, the magnet's attractive force is equal to the gravitational force of the suspended object. Then, based on the principle that the magnetic force is inversely proportional to the square of the gap between the magnet and the ferromagnetic body ${ }^{(17)}$, the actuator controls the air gap between the magnet and the object so as to adjust the attractive force. When the air gap is smaller than the balance gap, the actuator raises the magnet to increase the air gap; when the air gap is larger than the balance gap, the actuator lowers the magnet to decrease the air gap. In this way, the suspended object is levitated steadily without contact.

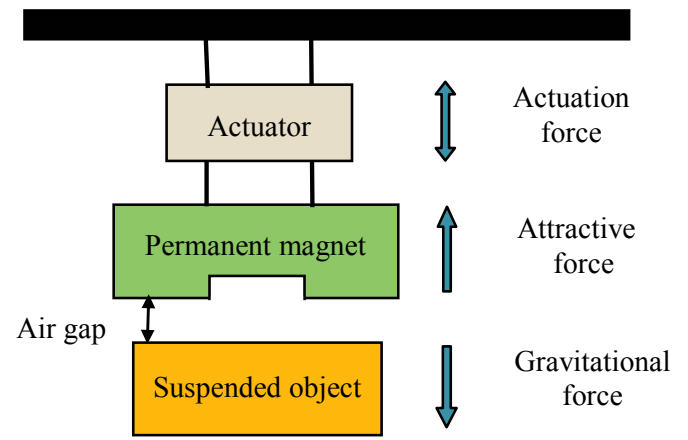

Fig. 1 A suspension system scheme

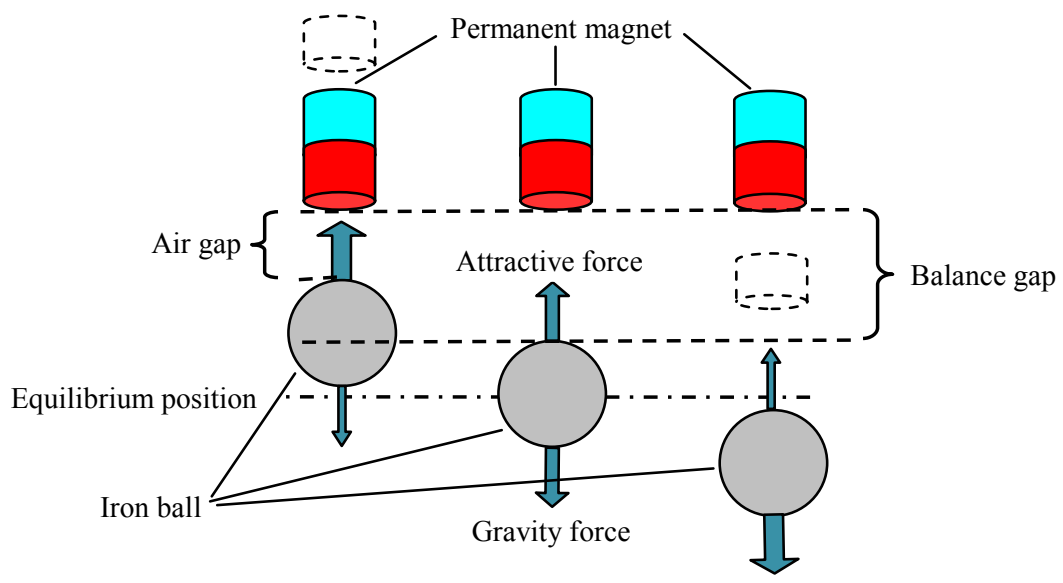

Fig. 2 Relationship between attractive force and gravitational force 


\section{Realization of zero power control}

\subsection{Zero power control in experimental prototype}

A photograph of the prototype of the permanent magnetic suspension device is shown in Fig. 3. Figure 4 shows the configuration of this magnetic suspension system. The prototype principally consists of a permanent magnet, a VCM, a spring, and two eddy current displacement sensors. The whole device has three parts: the suspended object is an iron ball whose diameter is $0.03 \mathrm{~m}$; the moving part consists of a permanent magnet, a slider of VCM, and a sensor target; and the base part consists of the VCM stator, two sensors, and the frame. The weight of the iron ball is $0.1098 \mathrm{~kg}$, and that of the moving part is $0.1478 \mathrm{~kg}$. The VCM used in this magnetic suspension prototype has a driving length of $0.015 \mathrm{~m}$ and a maximum generating force of $20 \mathrm{~N}$ at a coil current of $2 \mathrm{~A}$. The VCM is the only active driving element that is used to control the device suspension stably in the equilibrium. The two sensors are eddy current displacement sensors. The upper sensor measures the permanent magnet's position and has a resolution of $2 \times 10^{-5} \mathrm{~m}$ and a measurement range of $0.01 \mathrm{~m}$. The lower sensor measures the iron ball's position and has a resolution of $1 \times 10^{-6} \mathrm{~m}$ and a measurement range of $0.004 \mathrm{~m}$.

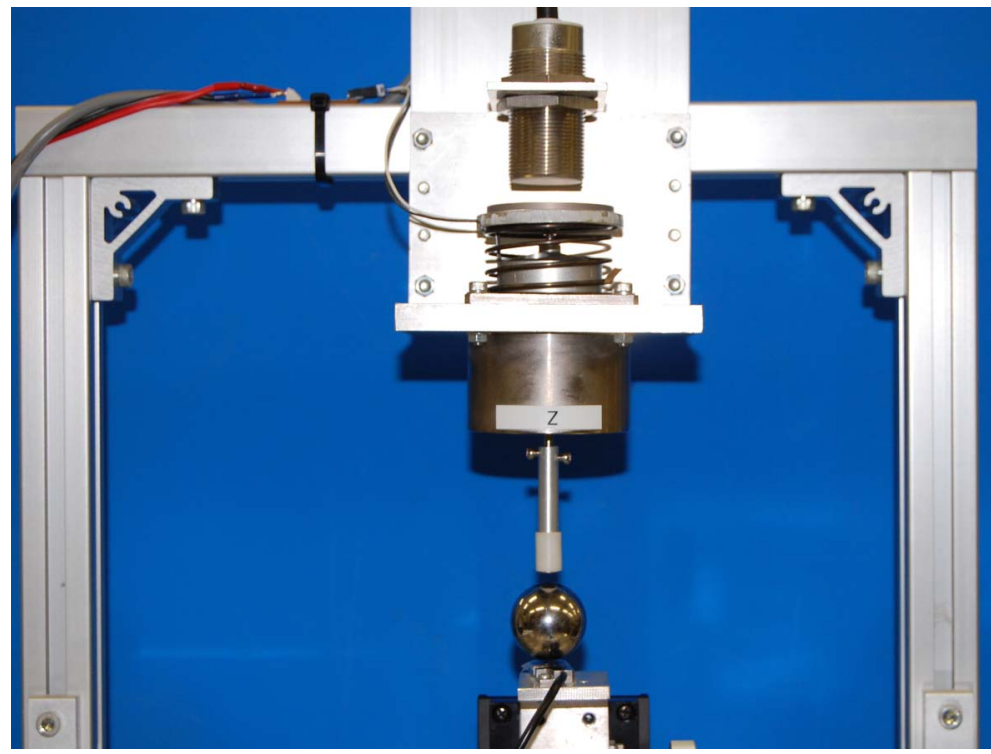

Fig. 3 Photograph of experimental prototype

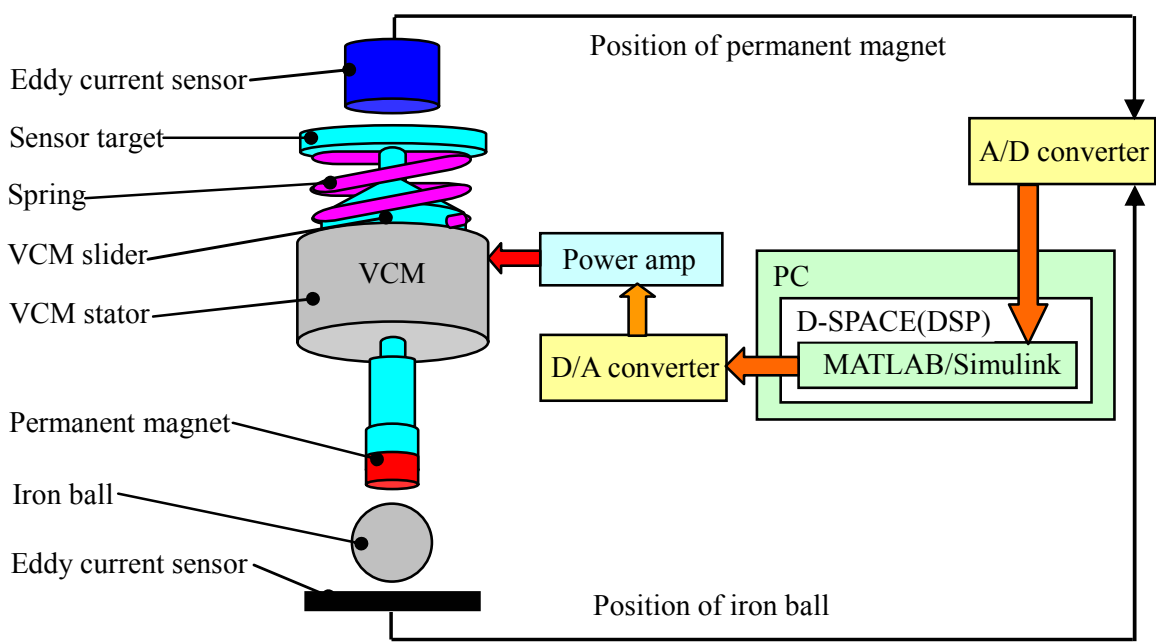

Fig. 4 Configuration of magnetic suspension system 
As shown in Fig. 4, in order to obtain the zero power control, a spring is installed between the VCM stator and the sensor target in the device. When the iron ball is steadily suspended, the gravitational force of the moving part and the iron ball must be transmitted to the base part. If there is no spring in the device, the force must be transmitted through the VCM, which means that the VCM normal force equals the gravitational force of the moving part and the iron ball. However, if there is a spring in the device, the spring's force can counterbalance the gravitational force acting on the moving part and the iron ball in the balanced state, and the VCM force can reach zero.

The controller of this suspension system is a DSP controller, as shown in Fig. 4. Signals from two sensors are input to the DSP controller through 12-bit A/D converters. Based on these signals, appropriate current values are computed by the controller. The current values are converted to analog current by D/A converters, and then amplified by a current amplifier. The final actual current controls the VCM to steadily maintain suspension of the ball.

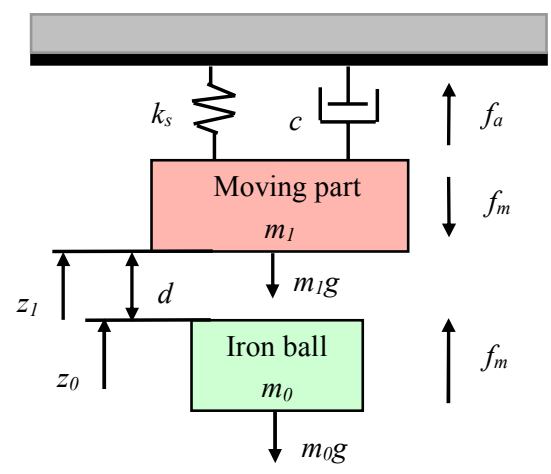

Fig. 5 Model of suspension system

\subsection{Zero power control in model}

A model of this permanent magnetic suspension system as shown in Fig. 5 has been set up for feasibility studies, stability confirmations, numerical simulations, and feedback gains calculations. According to the model, the equations of motion for the moving part and the suspended object are, respectively,

$$
\begin{aligned}
& m_{0} \ddot{z}_{0}=f_{m}-m_{0} g, \text { and } \\
& m_{1} \ddot{z}_{1}=-c \dot{z}_{1}-k_{s} z_{1}+f_{a}-f_{m}-m_{1} g
\end{aligned}
$$

In these equations, $m_{0}, m_{l}$ are the mass of the iron ball and the moving part; $z_{0}, z_{l}$, whose positive directions are up, are the displacements of the iron ball and the moving part; $f_{a}$ is the generating force of VCM; $f_{m}$ is the attractive force of the permanent magnet; $k_{s}$ is the spring constant; and $c$ is the damping coefficient including spring fading characteristics, VCM bearings frictions, and air viscosities.

Additionally, the relationship between the input current and the VCM generating force is represented by the equation

$$
f_{a}=k_{t} i
$$

where $k_{t}$ is the VCM propulsive coefficient and $i$ is the VCM input current.

The relationship between the permanent magnet attractive force and the air gap distance is described by

$$
f_{m}=\frac{k_{m}}{d^{2}}
$$


where $k_{m}$ is the coefficient of magnetic attractive forces and $d$ is the air gap distance between the permanent magnet and the iron ball.

According to Eqs. (1) and (2), when the iron ball is suspended in a static state, the differential terms become zero, i.e., the acceleration terms and the damping force become zero. If there is no spring in the device, the spring force is zero. Consequently, in order to maintain the balance, the VCM force has to equal the gravitational force of the moving part and the iron ball. During this time, the VCM current can never be zero. However, if there is a spring in the device, the spring force and the VCM force together afford the gravitational force acting on the moving part and the iron ball. Therefore, the VCM current can become zero in the static state. This means that the zero power control is mathematically feasible.

\subsection{Zero power control in controller}

There are many ways to realize zero power control in the magnetic suspension system controller, such as using an observer, a current integral feedback, or object weight estimation. In this paper, the current integral feedback method is investigated.

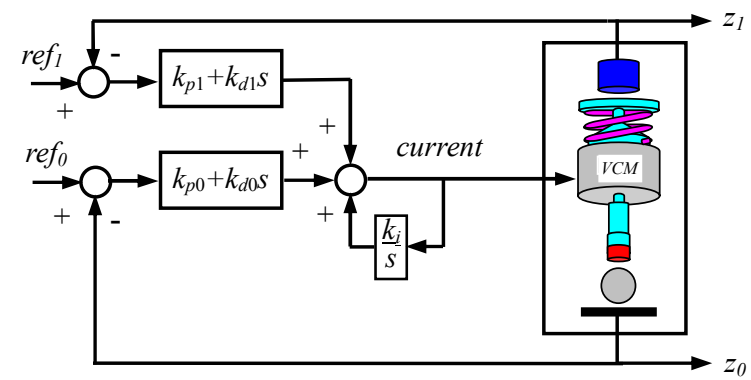

Fig. 6 Block diagram of control system

As shown in Fig. 6, the block diagram is the controller of the permanent magnetic suspension system, having an integral current feedback loop for zero power control. There are two large PD feedback loops. The lower PD feedback loop measures the position of the iron ball. The upper PD feedback loop observes the position of the permanent magnet. Both PD feedback loops calculate the current values based on the sensors' signals in order to make the VCM robustly maintain the system levitation. In addition to the two large PD feedback loops, a local current integral feedback loop is used before the levitated object block to implement zero power control. When current flows through the VCM circuit, this integral feedback loop reduces the current. The current reduction diminishes the generating force of the VCM, and the resulting variation causes the spring length and the generating force to change. Finally, when the spring force equals the gravitational force of the moving part and the iron ball, the VCM current reaches zero. Furthermore, the $k_{i}$ value for the gain of the integral current feedback loop influences the speed of current variation. If the value is large, the current reaches zero quickly. However, that works against system stability. Rapid current variation will result in system instability. Therefore, a proper $k_{i}$ value is very important for system stability.

\section{Results of numerical simulation}

\subsection{Simulation conditions}

In this feasibility study, numerical simulations were carried out with a nonlinear attractive force. The simulations were examined under varied conditions, such as the magnetic suspension system without springs and without zero power control, with springs and without zero power control, with springs and with zero power control, and using different springs and different $k_{i}$ gains. 
When the iron ball was levitated, a $-5 \times 10^{-5} \mathrm{~m}$ step value was added to the iron ball. After the step was added, the VCM current, the iron ball displacements, and the moving part displacements were recorded. The step was added at 1.5 seconds, and the responses were recorded until 5 seconds. The varieties of current and displacement were plotted, with the upward direction as positive.

Moreover, all the feedback gains used here were calculated using the LQR (linear quadratic regulator) full state feedback control law with a linear mathematical model.

\subsection{Simulation results}

The results of simulation without springs and without zero power control are shown in Fig. 7. The upper plot shows the VCM current response, the middle one indicates the iron ball displacement, and the lower one shows the moving part displacement. When the iron ball was levitated in a stable condition, before adding the step, the current was approximately $-0.258 \mathrm{~A}$, which was used to generate the force counterbalancing the gravitational force on the moving part and the iron ball. The negative current caused the VCM to generate an upward force. When the step was added, the iron ball moved
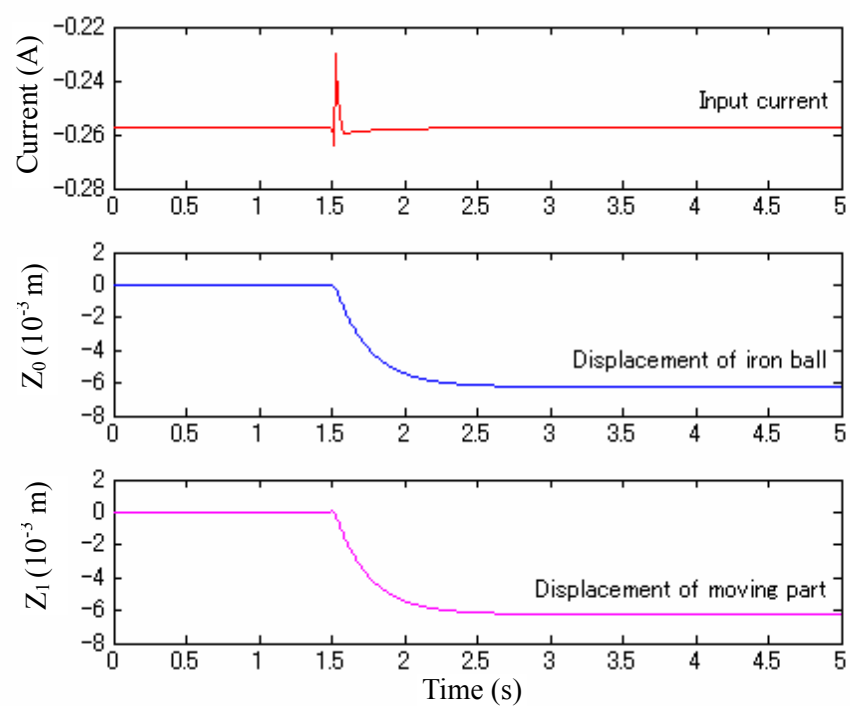

Fig. 7 Simulation results without springs and without zero power control
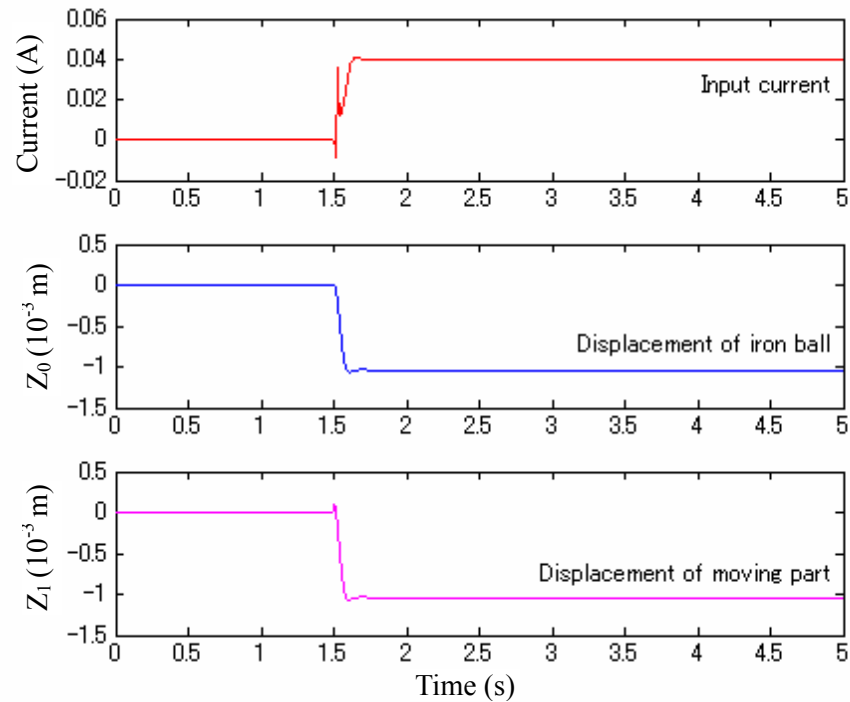

Fig. 8 Simulation results with springs and without zero power control Spring constant $k_{s}=400 \mathrm{~N} / \mathrm{m}$ 

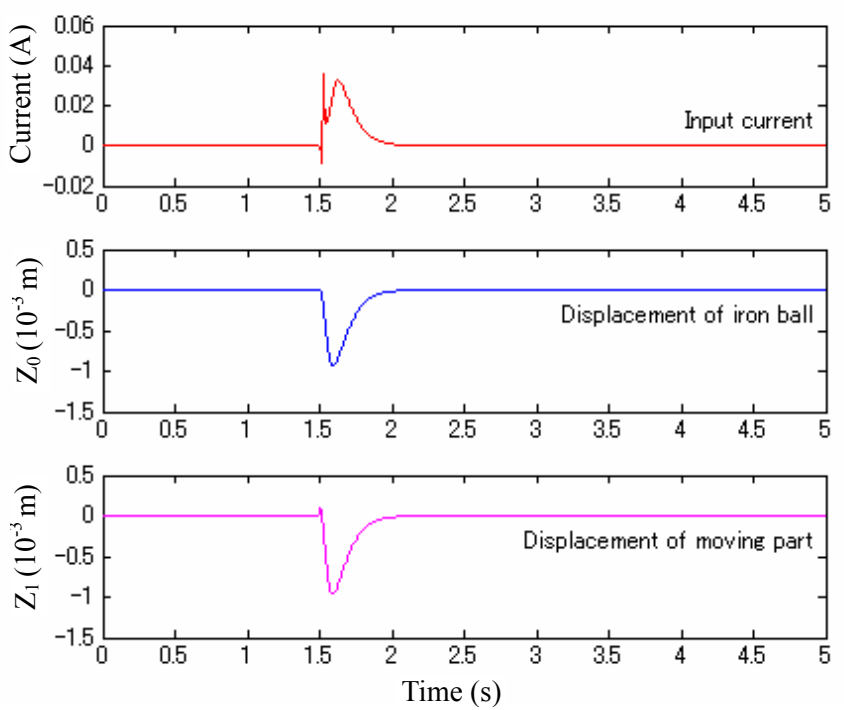

Fig. 9 Simulation results with springs and with zero power control Spring constant $k_{s}=400 \mathrm{~N} / \mathrm{m}$, integrator gain $k_{i}=6$

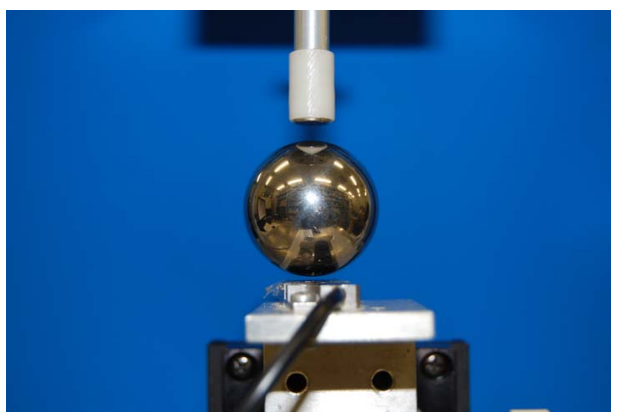

Fig. 10 Photograph of suspended iron ball

downward as shown in the middle graph, the air gap length between the magnet and the iron ball increased, and the attractive force of the magnet decreased. In order to make the levitation return to equilibrium, the actuator drove the magnet lower to reduce the air gap between the magnet and the iron ball, and then the attractive force increased until it was equal to the gravitational force. Thereafter, the levitation reached the balanced state again. Moreover, the VCM current after equilibrating was the same as that in the original equilibrium state since the generating force did not change.

The results of the simulation with springs and without zero power control are shown in Fig. 8. In this simulation, the spring constant used was $400 \mathrm{~N} / \mathrm{m}$. After adding the step, the current value was approximately $0.04 \mathrm{~A}$, which generated a downward force to counterbalance the spring force. The value was smaller than that shown in Fig. 5. In that moment, the spring force was equal to the summations of the VCM force and the gravitational forces of the iron ball and the moving part. In the changing progress, there was a sharp variation in the current because the VCM responded to the step, and subsequently changed the variation resulting from the spring's actions.

The results of the simulation with springs and with zero power control are shown in Fig. 9. In this simulation, except for the current integral feedback loop, all of the conditions and feedback gains were the same as in the simulation shown in Fig. 8. As shown in Fig. 9, after adding the step and reaching the equilibrium state, the current and the displacements of the iron ball and the moving part returned to zero. This result indicates that in the simulation with spring and with zero power control, the VCM consumed no energy and the spring force counterbalanced all the gravitational forces in the equilibrium state.

All numerical simulation results imply that the zero power control using a spring and 
an integral current feedback loop is feasible in this suspension system with a permanent magnet motion feedback loop.

\section{Experimental results}

Non-contact suspension experiments were also implemented using the experimental prototype shown in Fig. 3. Figure 10 shows an iron ball levitating in a stable state. Experiments under various conditions and corresponding to the numerical simulations were completed. However, on account of the upper sensor in the experimental prototype having poor linearity, the feedback gains used in the experiments differed from those used in the simulations. Some experimental results are shown here, and all the current results in the figures were passed through low pass filters.

The results of the experiment without springs and without zero power control are shown in Fig. 11. From these results, it can be seen that when the iron ball was suspended in a stable state, the VCM current was approximately $-0.26 \mathrm{~A}$, which generated a force counterbalancing the gravitational force of the iron ball and the moving part. After adding the step value, the iron ball and the moving part moved downward together, and the current
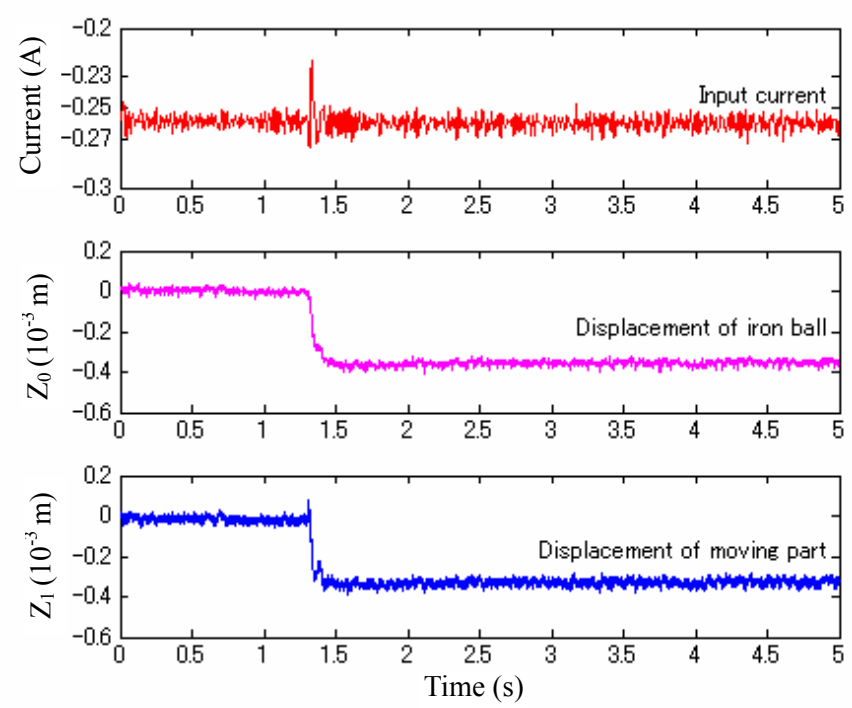

Fig. 11 Experimental results without springs and without zero power control
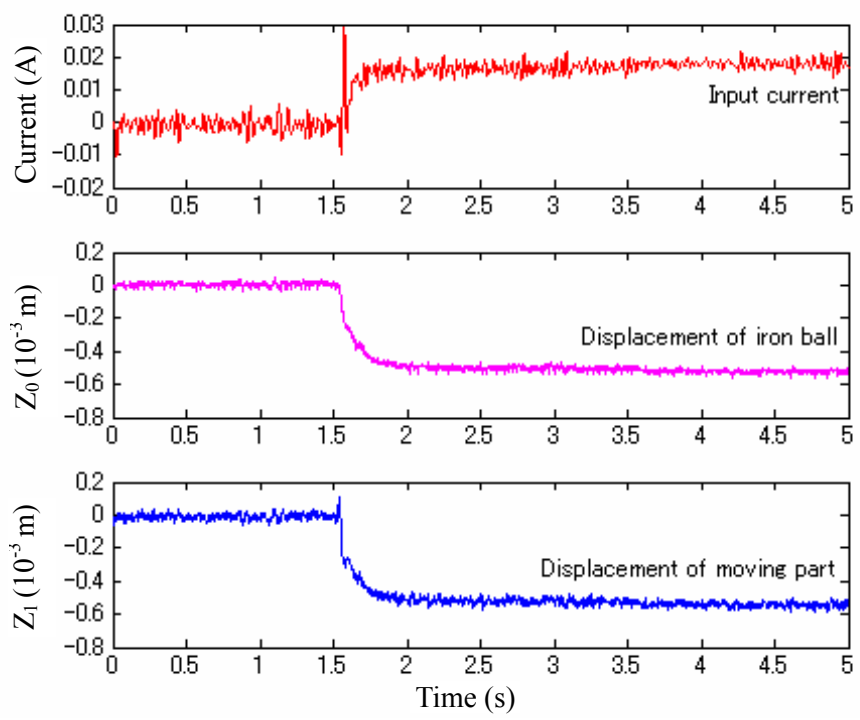

Fig. 12 Experimental results with springs and without zero power control Spring constant $k_{s}=400 \mathrm{~N} / \mathrm{m}$ 
returned to the original value, in agreement with the simulation results. The step only changed the position of the iron ball but did not change the loads; therefore, the current did not change. Figure 11 indicates that the VCM current in the normal suspension state was approximately $-0.26 \mathrm{~A}$.

Figure 12 shows the experimental results with springs and without zero power control. The spring constant was $400 \mathrm{~N} / \mathrm{m}$. In the current graph in Fig. 12, it can be seen that after adding the step, the current value was approximately $0.02 \mathrm{~A}$ in the stable state. This was much smaller than the current in Fig. 11.

Figure 13 shows the experimental results with springs and with zero power control. As shown in Fig. 13, the current and the displacements all converged to zero in the stable state. The current integral feedback loop caused all the variation. The integral loop reduced the VCM input current to zero, and then the VCM generated no force. Therefore, the spring maintained all of the gravitational forces, and the iron ball and the moving part returned to their original positions.

Figure 14 and Figure 15 show the experimental results using a different spring whose
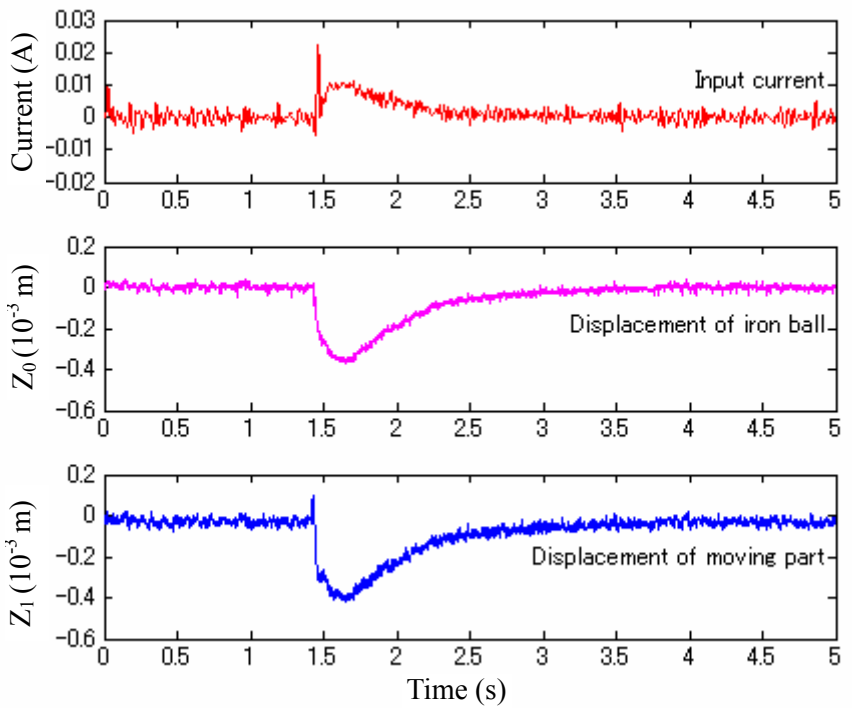

Fig. 13 Experimental results with springs and with zero power control Spring constant $k_{s}=400 \mathrm{~N} / \mathrm{m}$, integrator gain $k_{i}=6$
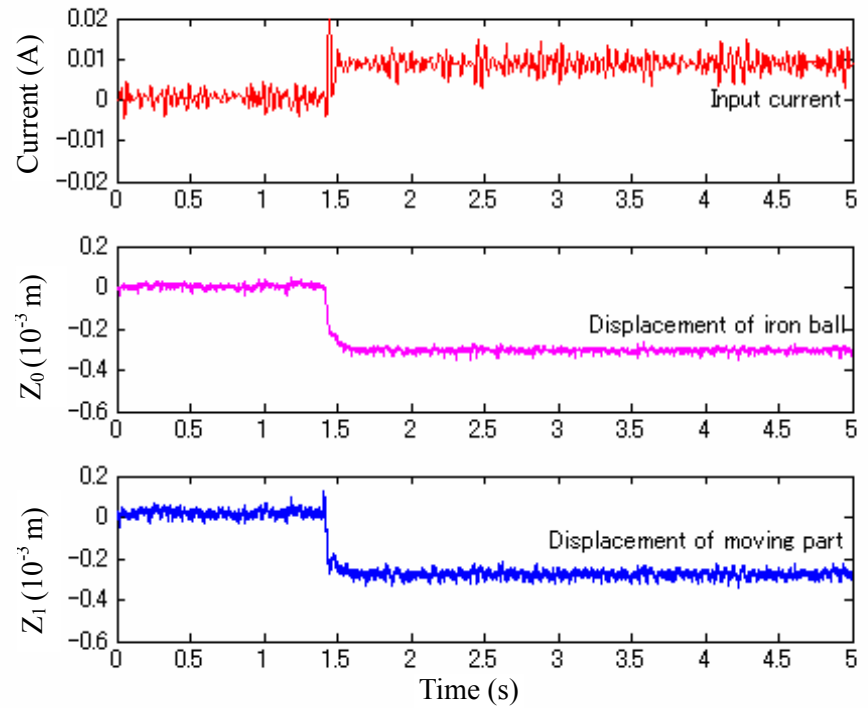

Fig. 14 Experimental results with springs and without zero power control Spring constant $k_{s}=300 \mathrm{~N} / \mathrm{m}$ 

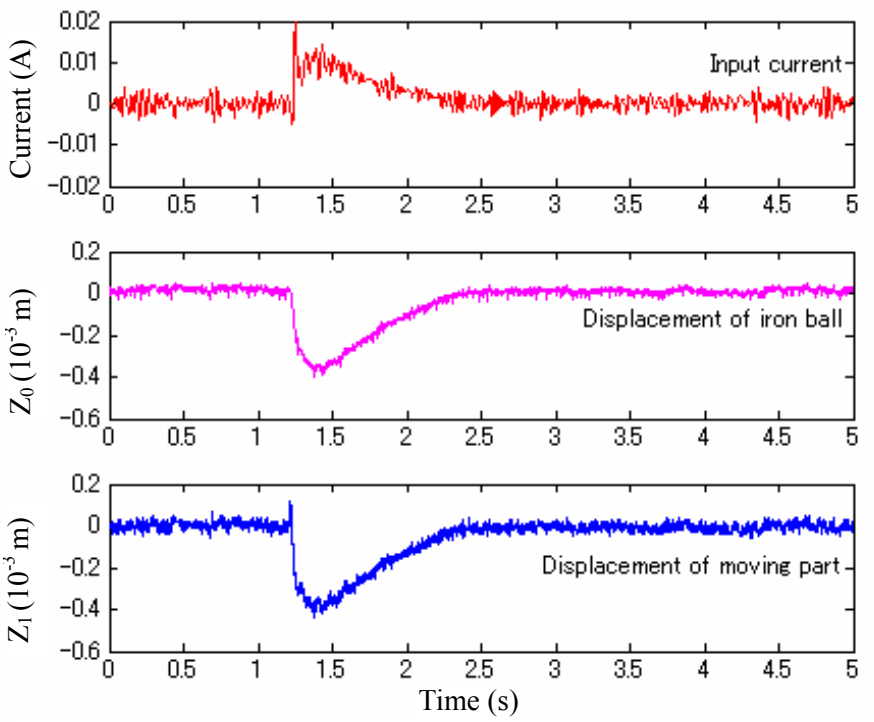

Fig. 15 Experimental results with springs and with zero power control Spring constant $k_{s}=300 \mathrm{~N} / \mathrm{m}$, integrator gain $k_{i}=6$

spring constant was $300 \mathrm{~N} / \mathrm{m}$. The experiments were carried out with the same feedback gains and conditions of Figs. 12 and 13. As shown in Fig. 14, after adding the step, the current was approximately $0.01 \mathrm{~A}$ in the stable state, which was smaller than that in Fig. 12. This was because weak springs require small forces to counterbalance. Moreover, the current and displacements in Fig. 15 converged to zero as well.

In conclusion, the experimental and simulation results are very similar, and they suggest that the zero power control can be successfully applied in this suspension system with a permanent magnet motion feedback loop.

\section{Conclusions}

A zero power control method was proposed for used in a permanent magnetic suspension system. Based on the analysis of the feasibilities of zero power control in an experimental prototype, in a model, and in a controller and on the results of the numerical simulations and experiments, the following conclusions can be drawn.

First, through the use of a digital controller with two PD feedback loops, the suspension system with the permanent magnet and linear actuator can be levitated steadily without contact.

Secondly, the zero power control method using a spring in suspension device and a current integral feedback loop in the controller can reduce energy consumption considerably in this suspension system with a permanent magnet motion feedback loop.

\section{References}

(1) Morishita, M. and Azukizawa, T., Zero Power Control Method for Electromagnetic Levitation System (in Japanese), Trans. IEE of Japan, Vol.108-D, No.45 (1988), p.447

(2) Misuno, T. and Yoshitomi, R., Vibration Isolation System Using Zero-Power Magnetic Suspension (1st Report, Principle and Basic Experiments) (in Japanese), Transactions of the Japan Society of Mechanical Engineers, Series C, Vol.68, No.673 (2002), pp. 2599-2604

(3) Misuno, T., Kishita, D., Takasaki, M. and Ishino, Y., Vibration Isolation System Using Zero-Power Magnetic Suspension (2st Report, Introduction of Weight Support Mechanism) (in Japanese), Transactions of the Japan Society of Mechanical Engineers, Series C, Vol.72, No.715 (2006), pp. 714-722

(4) Ishino, Y., Misuno, T. and Takasaki, M., In Creasing the Load Capacity of Magnetic Suspension by a Stiffness Switching Control (in Japanese), Dynamics and Design Conference 2008, No.08-14 (2008. 9), p.658 
(5) Kim, Y.H., Kim, K.M. and Lee, J., Zero Power Control with Load Observer in Controlled-PM Levitation, IEEE Transactions on Magnetics, Vol.37, No.4 (2001), pp.2851-2854

(6) Morishita, M. and Iton, H., The Self-gap-detecting Zero Power Controlled Electromagnetic Suspension System (in Japanese), Trans. IEE of Japan, Serias D, Vol.126, No.12 (2006), pp.1667-1677

(7) Non, M.D., Antaki, J, F., Ricci, M., Gardiner, J., Paden, D., Wu, J.C., Prem, E., Borovetz, H. and Paden, B.E., Magnetic Design for the PediaFlow Ventricular Assist Device, Artificial Organs, Vol.32, No.2 (2007), pp.127-135

(8) Misuno, T., Hirai, Y., Ishino, Y. and Misuno, T., Flux-Path Control Magnetic Suspension System Using Voice Coil Motors, Journal of System Design and Dynamics, Vol.1, No.2 (2007), pp.147-158

(9) Ueno, T. and Higuchi, T., Zero-Power Magnetic Levitation Using Composite of Magnetostrictive/Piezoelectric Material, IEEE Transactions on Magnetics, Vol.437, No.8 (2007), pp.3477-3482

(10) Oka, K., Ninomiya, N., Chen, L. and Fujiwara, Y., Magnetic Suspension System with Variable Flux Path Mechanism Using Rotary Actuator, Tenth International Symposium on Magnetic Bearings2006, p.86

(11) Oka, K. and Cui, T.S., Zero Power Control for Magnetic Suspension System with Permanent Magnet Motion Control, International Conference on Electrical Machines and Systems, CD-ROM,2004

(12) Oka, K. and Cui, T.S., 2 DOF Suspension Mechanism for Noncontact Manipulation with Permanent Magnet Motion Control, Proc. of Mechatronics and Robotics 2004, pp.383-387

(13) Oka, K., Noncontact Manipulation with Permanent Magnet Motion Control, Proceeding of the 4th International Symposium on Linear Drivers for Industry Applications, pp.259-262

(14) Cui, T.S., Fujiwara, Y. and Oka, K., 2 DOF Maglev System with Permanent Magnet Motion Control, SICE Annual Conference 2005, pp.3859-3862

(15) Oka, K., Fujiwara, Y., Cui, T.S. and Chen, L., Noncontact Spinning Mechanism Using Linearly Actuated Magnets, Proc. of the 5th International Symposium on Linear Drivers for Industry Applications 2005, pp.552-555

(16) Fujiwara, Y., Cui, T.S. and Oka, K., Magnetic Levitation System with Permanent Magnet Motion Control (Study of Spinning Mechanism) (in Japanese), Dynamics and Design Conference 2008, No.04-5 (2004. 9), p.517

(17) Shameli, E., Khamesee, M.B. and Huissoon, J.P., Nonlinear Controller Design for a Magnetic Levitation Device, Microsyst Techno, No.13 (2007), pp.831-83 